\title{
Appraising growth differentiation factor 15 as a promising biomarker in digestive system tumors: a meta-analysis
}

\author{
Yanqiu Wang ${ }^{1 *}$, Tao Jiang ${ }^{1}$, Mingyan Jiang ${ }^{2}$ and Shuijing Gu ${ }^{3}$
}

\begin{abstract}
Background: Previous studies have highlighted cytokine growth differentiation factor 15 (GDF-15) as a potential biomarker for digestive system tumors (DST). This study sought to assess the feasibility of using GDF-15 as a diagnostic and prognostic biomarker in DST.

Methods: Eligible studies from multiple online databases were reviewed. Meta-analyses of diagnostic parameters were carried out using standard statistical methods. Study-specific hazard ratios (HRs) with 95\% confidence intervals (Cls) were calculated to estimate the strength of the relationship between GDF-15 levels and clinical prognosis.

Results: We identified 17 eligible studies comprising 3966 patients with DST. The sensitivity, specificity, and area under the curve (AUC) for the discriminative performance of GDF-15 as a diagnostic biomarker were 0.74 (95\% Cl: 0.68-0.80), 0 . 83 (95\% Cl: 0.75-0.89), and 0.84, respectively. Moreover, increased GDF-15 expression levels were markedly associated with unfavorable overall survival (OS) in patients with DST (HR=2.34, 95\% Cl: $\left.2.03-2.70, P<0.001 ;\left.\right|^{2}=0.0 \%\right)$ and colorectal cancer (CRC) (HR=2.27, 95\% Cl: 1.96-2.63, $\left.P<0.001 ; 1^{2}=0.0 \%\right)$. Stratification by cancer type, test matrix, ethnicity, and cutoff setting also illustrated the robustness of the diagnostic value of GDF-15 in DST.
\end{abstract}

Conclusion: Collectively, our data suggest that GDF-15 expression level may have value as a diagnostic and prognostic biomarker, independent of other, traditional biomarkers.

Keywords: Cytokine growth differentiation factor 15, Digestive system tumor, Diagnosis, Prognosis, Meta-analysis

\section{Background}

Over the past decade, digestive system tumors (DST) have become major causes of cancer-related mortality worldwide [1]. According to global cancer statistics compiled in 2016, death rates have increased for patients with DST, including for those with liver cancer and pancreatic cancer [2]. Due to lack of sensitive diagnostic testing, large numbers of patients with DST are mostly diagnosed at advanced stages, resulting in poor 5-year survival rates [2]. It is therefore necessary to identify novel, reliable biomarkers which can predict early diagnosis and/or prognosis of patients with DST.

Human growth differentiation factor 15 (GDF-15), also known as macrophage inhibitory cytokine-1 (MIC-1), is

\footnotetext{
* Correspondence: www926sci@163.com

'Department of Clinical Laboratory, The First Affiliated Hospital, and College of Clinical Medicine of Henan University of Science and Technology, No.24 Jinghua Road, Jianxi District, Luoyang 471000, China

Full list of author information is available at the end of the article
}

a divergent member of the transforming growth factor- $\beta$ (TGF- $\beta 1$ ) superfamily of proteins [3, 4]. The human GDF-15 gene maps to chromosome 19 in the p13.113.2 region, and encodes a $25-\mathrm{kDa}$ secreted growth factor that is highly expressed in cardiomyocytes, adipocytes, endothelial cells, and macrophages in both normal and diseased tissues [3-5]. Intriguingly, GDF-15 levels are substantially increased in various pathological conditions, including inflammation and injury [5-7]. Notably, experimental and epidemiological evidence has demonstrated that GDF-15 levels are up-regulated in many types of DST, such as colorectal cancer (CRC) [8-13], gastrointestinal cancer (GC) $[14,15]$, pancreatic cancer (PC) [16-20], esophageal carcinoma (EC) [21, 22], and liver cancer [23, 24]. Recently, GDF-15 has received much attention as a diagnostic and prognostic biomarker in DST. However, data are inconsistent among studies assessing the clinical relevance of GDF-15, and

(c) The Author(s). 2019 Open Access This article is distributed under the terms of the Creative Commons Attribution 4.0 International License (http://creativecommons.org/licenses/by/4.0/), which permits unrestricted use, distribution, and reproduction in any medium, provided you give appropriate credit to the original author(s) and the source, provide a link to the Creative Commons license, and indicate if changes were made. The Creative Commons Public Domain Dedication waiver (http://creativecommons.org/publicdomain/zero/1.0/) applies to the data made available in this article, unless otherwise stated. 
the statistical power of these studies has also been insufficient. In this study, we collected published studies regarding the expression of GDF-15 in DST and performed a meta-analysis to determine whether high GDF-15 expression levels can be used as a diagnostic or prognostic biomarker in DST.

\section{Methods}

\section{Literature search}

We searched the PubMed, EMBASE, ESBCO, Wiley Online Library, and Ovid databases for eligible studies from their incipience to June 20, 2018. We used the following search terms or Medical Subject Headings $(\mathrm{MeSH})$ words to identify eligible studies: "macrophage inhibitory cytokine-1/MIC-1/growth differentiation factor 15/GDF-15" AND "oesophageal cancer/oesophageal neoplasm/colorectal cancer/colorectal carcinoma/colon cancer/colon carcinoma/CRC /gastrointestinal cancer/gastric carcinoma/gastric cancer/stomach cancer/hepatocellular carcinoma/liver cancer/pancreatic carcinoma/ pancreatic neoplasms/pancreatic ductal adenocarcinoma/ pancreatic mass/digestive system tumor/digestive system neoplasm" AND "survival/prognosis/outcome/hazard ratio/ HR" OR "diagnosis/sensitivity/specificity/ROC/AUC/area under the curve". Reference lists of the included articles or relevant reviews were also browsed for potentially missing studies.

\section{Inclusion and exclusion criteria}

Studies meeting the following criteria were included: (1) clinical trials reporting the diagnostic and/or prognostic features of GDF-15 in DST; (2) studies where the diagnostic parameters or survival outcomes included sensitivity, specificity, area under the curve (AUC), overall survival (OS), disease free survival (DFS), progression-free survival (PFS), recurrence-free survival (RFS), tumor-specific survival (TSS), or cancer-specific survival (CSS); and (3) the estimated hazard ratios (HR) or odds ratio (OR) with corresponding 95\% confidence intervals (CIs) were available or could be calculated from published data. Accordingly, exclusion criteria included: (1) studies defined as reviews, basic studies, animal studies, letters, or conference abstracts; (2) data for statistical analyses were unavailable, and also failed to contact the authors; (3) studies with high risk and bias in quality assessment; and (4) articles written in a language other than English.

\section{Data extraction and quality assessment}

Data extraction was performed for study sensitivity, specificity, sample numbers, as well as HRs and their corresponding 95\% CIs. Where such data were unavailable, the values were calculated indirectly using Engauge Digitizer 4.1 software. Other information included the first author's name, article date, patient ethnicity, specimen type, test method, cut-off value settings, survival points, follow-up time, quantiles of GDF-15, and other relevant clinicopathological characteristics.

Study quality was judged according to the Quality Assessment of Diagnosis Accuracy Studies criteria (QUADAS), which is based on a 14-item list [25]. The quality of all retrospective cohort studies was assessed using the Newcastle-Ottawa Scale (NOS) checklist, wherein potential bias due to cohort selection, comparability, and outcome ascertainment is judged on a score ranging from 0 to 9 [26]. The included studies were eliminated if they were scored to be of low quality (i.e. a final score of less than 5 for NOS or 8 for Quality Assessment of Diagnostic Accuracy Studies [QUADAS]).

\section{Statistical analysis}

Statistical analyses were conducted using STATA 12.0 software (Stata Corporation, College Station, TX, USA). The primary outcomes (pooled sensitivity, specificity, positive likelihood ratio (PLR), negative likelihood ratio (NLR), diagnostic odds ratio (DOR), and AUC with corresponding 95\% CIs) were obtained in the diagnostic meta-analysis; a pooled HR with 95\% CI was calculated to measure the association between GDF-15 expression (high vs. low) and the clinical outcomes of patients with DST. A combined HR $>1$ implied that GDF-15 had a negative effect on the survival outcome of the patients. Heterogeneity for the size of each effect was calculated using Cochran's $\mathrm{Q}$ and $\mathrm{I}^{2}$ statistics, and with statistical significance defined as $P<0.05$ or $\mathrm{I}^{2}>50 \%$. Fixed or random meta-analysis models were selected depending on the degree of study heterogeneity. Influence analysis was undertaken to ascertain the effects of outlier studies on the overall results. Publication bias was examined using Deek's funnel plot asymmetry test, as well as Egger's and Begg's tests, with statistical significance defined as $P<$ 0.05 .

\section{Results}

Search results and study quality

Figure 1 schematically displays the selection procedure for eligible articles. According to our search criteria, a total of 3281 studies were eligible after the elimination of duplicates among databases. Among them, 3217 records were excluded due to irrelevant content or non-original data after reading the titles and abstracts. In the subsequent stages of study selection, 64 studies were assessed based on full-text evaluation, and with another 47 excluded. Finally, 17 articles (12 relating to diagnosis, and 9 relating to prognosis) were included in the final meta-analysis.

Study bias judged, as by the 14-item QUADAS list or NOS checklist, revealed that all of the diagnostic and prognostic studies had QUADAS scores of $\geq 10$ 


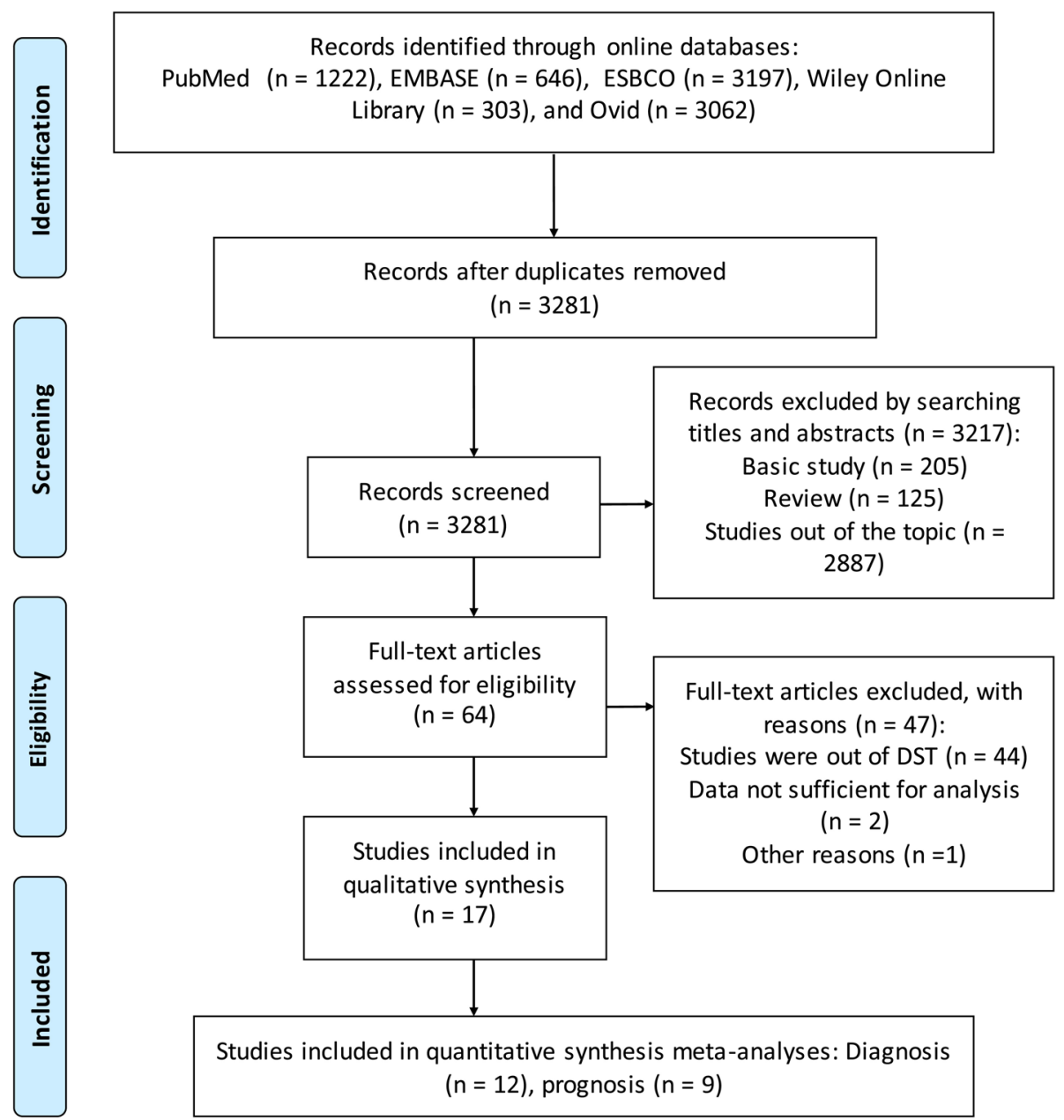

Fig. 1 Flow diagram of the study selection and exclusion criteria

or NOS scores of $\geq 6$ (Table 1 , Additional file 1 and Additional file 2), indicating that these data were suitable for our final statistical analysis.

\section{Study characteristics}

The main features of the included studies relating to the diagnostic role of GDF-15 are displayed in Table 1. Twelve diagnostic studies [12, 13, 15-24], comprising 2380 patients and 4630 paired controls, were included in the diagnostic meta-analysis. The study participants involved included Chinese [12, 13, 16, 20, 21, 23, 24], Australian [17, 19, 22], American [18], Polish [20], and Spanish [15] patients, with sample sizes ranging from 42 to 807 . The types of DST covered in these studies included colorectal cancer (CRC) [8-13], gastrointestinal cancer (GC) [14, 15], pancreatic cancer (PC) [16-19], esophageal carcinoma (EC) [21, 22], and liver cancer $[23,24]$, of which the final diagnoses were all confirmed histologically by surgical operation. The types of samples collected included plasma [18, 22], serum $[12,13,15-17,19,21,23,24]$, and tissue [22] samples obtained prior to treatment. Enzyme linked immunosorbent assay (ELISA) was primarily used to test for GDF-15 levels [12, 13, 15-19, 21-24], and only one study used multiplexed tandem PCR (MT-PCR) [22].

We also included 9 cohort studies [8-11, 13-15, 21, 22], with a total sample size of 2200 , to assess the association between GDF-15 expression levels (high vs. low) and the clinical outcomes of patients with DST (Table 2). All 9 studies were retrospective, and study populations included Chinese [8, 13, 21], Australian [10, 14, 22], American [11], and Spanish [15] patients. Survival outcomes included OS [8-11, 14, 15, 22], CSS or TSS [11, 13, 21], RFP [21], and PFS [15], with an average follow-up time of 30 months to 9.2 years. In one study [11], HRs calculated based on different quartile points were judged as independent data. However, survival outcomes like RFP and PFS were not combined due to insufficient study numbers. 


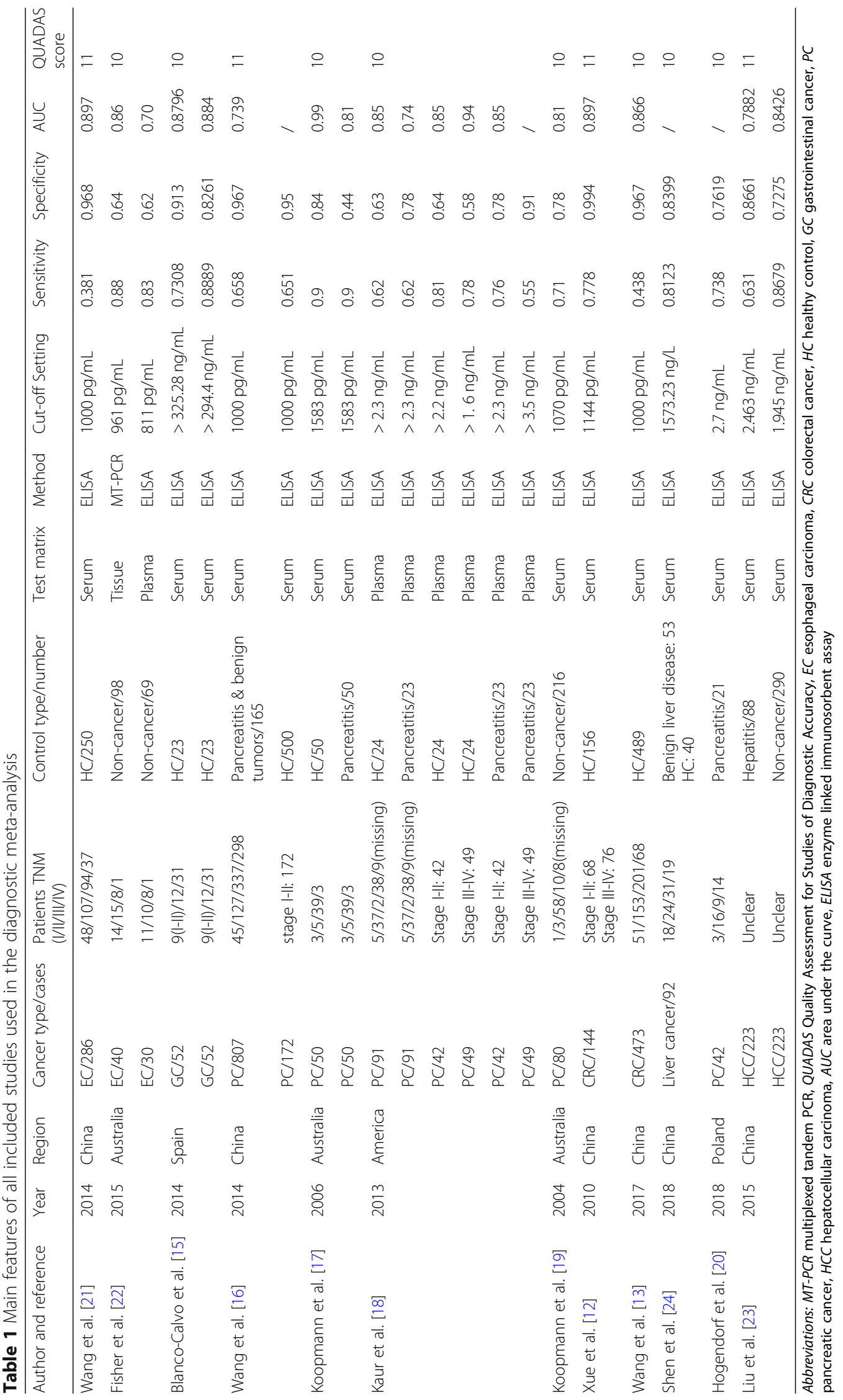


Table 2 Main features of all included studies used in the prognostic meta-analysis

\begin{tabular}{|c|c|c|c|c|c|c|c|c|c|c|c|c|c|c|}
\hline Author \& reference & Year & Country & $\begin{array}{l}\text { Cancer } \\
\text { type }\end{array}$ & $\begin{array}{l}\text { Patient } \\
\text { size }\end{array}$ & $\begin{array}{l}\text { Sample } \\
\text { type }\end{array}$ & Method & $\begin{array}{l}\text { Expression } \\
\text { level }\end{array}$ & $\mathrm{HR}$ & $\begin{array}{l}\text { Survival } \\
\text { point }\end{array}$ & $P$ value & $\begin{array}{l}\text { Follow-up } \\
\text { time }\end{array}$ & extraction & $\begin{array}{l}\text { Quartiles } \\
\text { of GDF-15 }\end{array}$ & $\begin{array}{l}\text { NOS } \\
\text { score }\end{array}$ \\
\hline Li et al. [8] & 2016 & China & CRC & 138 & Serum & ELISA & Increased & 1.915 & OS & 0.045 & Unclear & Directly & / & 6 \\
\hline \multirow[t]{2}{*}{ Wang et al. [13] } & \multirow[t]{2}{*}{2017} & \multirow[t]{2}{*}{ China } & \multirow[t]{2}{*}{ CRC } & \multirow[t]{2}{*}{94} & \multirow[t]{2}{*}{ Serum } & \multirow[t]{2}{*}{ ELISA } & \multirow[t]{2}{*}{ Increased } & 2.917 & TSS & 0.0005 & \multirow{2}{*}{$\begin{array}{l}\text { Average: } \\
43 \text { months }\end{array}$} & \multirow[t]{2}{*}{ Directly } & \multirow[t]{2}{*}{ / } & \multirow[t]{2}{*}{8} \\
\hline & & & & & & & & 2.607 & TSS & 0.007 & & & & \\
\hline Wallin et al. [9] & 2011 & Sweden & CRC & 320 & Plasma & SP-PLA & Increased & 2.11 & OS & 0.002 & $\begin{array}{l}\text { Median: } \\
6 \text { years }\end{array}$ & Directly & / & 8 \\
\hline Brown et al. [10] & 2003 & Australia & CRC & 261 & Serum & ELISA & Increased & 2.2 & OS & 0.0034 & 60 months & Directly & / & 8 \\
\hline \multirow[t]{18}{*}{ Mehta et al. [11] } & \multirow[t]{18}{*}{2015} & \multirow[t]{18}{*}{ America } & \multirow[t]{18}{*}{ CRC } & \multirow[t]{18}{*}{618} & \multirow[t]{18}{*}{ Plasma } & \multirow[t]{18}{*}{ ELISA } & Increased & 1.88 & OS & $<0.0001$ & \multirow{18}{*}{$\begin{array}{l}\text { Median: } \\
9.2 \text { years }\end{array}$} & \multirow[t]{18}{*}{ Directly } & \multirow[t]{6}{*}{ Quartile2 } & \multirow[t]{18}{*}{8} \\
\hline & & & & & & & Increased & 1.77 & OS & 0.0002 & & & & \\
\hline & & & & & & & Increased & 1.74 & OS & 0.0002 & & & & \\
\hline & & & & & & & Increased & 1.94 & CSS & 0.003 & & & & \\
\hline & & & & & & & Increased & 1.65 & CSS & 0.01 & & & & \\
\hline & & & & & & & Increased & 1.67 & CSS & 0.009 & & & & \\
\hline & & & & & & & Increased & 2.51 & OS & $<0.0001$ & & & \multirow[t]{6}{*}{ Quartile3 } & \\
\hline & & & & & & & Increased & 2.55 & OS & 0.0002 & & & & \\
\hline & & & & & & & Increased & 2.54 & OS & 0.0002 & & & & \\
\hline & & & & & & & Increased & 2.64 & CSS & 0.003 & & & & \\
\hline & & & & & & & Increased & 2.6 & CSS & 0.01 & & & & \\
\hline & & & & & & & Increased & 2.67 & CSS & 0.009 & & & & \\
\hline & & & & & & & Increased & 2.85 & OS & $<0.0001$ & & & \multirow[t]{6}{*}{ Quartile4 } & \\
\hline & & & & & & & Increased & 2.63 & OS & 0.0002 & & & & \\
\hline & & & & & & & Increased & 2.63 & OS & 0.0002 & & & & \\
\hline & & & & & & & Increased & 2.73 & CSS & 0.003 & & & & \\
\hline & & & & & & & Increased & 2.34 & CSS & 0.01 & & & & \\
\hline & & & & & & & Increased & 2.4 & CSS & 0.009 & & & & \\
\hline Fisher et al. [22] & 2015 & Australia & EC & 138 & Plasma & ELISA & Increased & 2.91 & OS & 0.076 & 60 months & Directly & / & 9 \\
\hline & & & & & & & & 3.87 & OS & 0.048 & & & & \\
\hline Wang et al. [21] & 2014 & China & ESCC & 286 & Serum & ELISA & Increased & 2.557 & TSS & 0.002 & Average: & Directly & / & 8 \\
\hline & & & & & & & & 2.625 & TSS & 0.005 & & & & \\
\hline & & & & & & & & 1.739 & RFS & 0.047 & & & & \\
\hline & & & & & & & & 1.789 & RFS & 0.050 & & & & \\
\hline $\begin{array}{l}\text { Skipworth } \\
\text { et al. [14] }\end{array}$ & 2010 & Australia & OGC & 293 & Plasma & ELISA & Increased & 1.549 & OS & 0.036 & $\begin{array}{l}\text { Over } 1500 \\
\text { days }\end{array}$ & Directly & / & 8 \\
\hline Blanco-Calvo & 2014 & Spain & GC & 52 & Serum & ELISA & Increased & 3.843 & OS & 0.001 & Median:118.9 & Directly & / & 8 \\
\hline & & & & & & & & 3.608 & PFS & $<0.001$ & & & & \\
\hline
\end{tabular}

Abbreviations: OGC oesophago-gastric cancer, ESCC esophageal squamous cell carcinoma, GC Gastrointestinal cancer, EC esophageal carcinoma, OS overall survival, DFS disease-free survival, PFS progression-free survival, TSS tumor-specific survival, CSS cancer-specific survival, ELISA enzyme linked immunosorbent assay, NOS Newcastle-Ottawa Scale checklist, $H R$ hazard ratio

\section{Heterogeneity}

In the diagnostic meta-analysis, heterogeneity was observed in the overall pooled data, of which the $\mathrm{I}^{2}$ value was estimated to be $99.38 \%(P<0.001)$. Heterogeneity was also detected among 6 groups in our collected diagnostic data (Table 3), with $\mathrm{I}^{2}$ values ranging from 78.4 to $93.7 \%(P<0.0001)$. Thus, random effect models were used for these studies. In our pooled data for prognosis, no significant heterogeneity was detected.

\section{Diagnostic meta-analyses}

The overall pooled sensitivity, specificity, diagnostic odds ratio (DOR), and area under the curve (AUC) for GDF-15, used to distinguish DST from non-cancerous tumors, were 0.74 (95\% CI: $0.68-0.80), 0.83$ (95\% CI: $0.75-0.89$ ), 14.07 (95\%CI: 9.12-21.71), and 0.84, respectively (Fig. 2 and Table 3), corresponding to a positive likelihood ratio (PLR) of 4.38 (95\%CI: 3.00-6.39) and a negative likelihood ratio (NLR) of 0.31 (95\%CI: 0.25 0.38 ). These results suggest that GDF-15 level is a useful 
Table 3 Subgroup analyses of GDF-15 testing for CRC based on different covariates

\begin{tabular}{|c|c|c|c|c|c|c|c|}
\hline Analysis & $\begin{array}{l}\text { Sensitivity } \\
{[95 \% \mathrm{Cl}]}\end{array}$ & $\begin{array}{l}\text { Specificity } \\
{[95 \% \mathrm{Cl}]}\end{array}$ & $\begin{array}{l}\text { PLR } \\
{[95 \% \mathrm{Cl}]}\end{array}$ & $\begin{array}{l}\text { NLR } \\
{[95 \% \mathrm{Cl}]}\end{array}$ & $\begin{array}{l}\text { DOR } \\
{[95 \% \mathrm{Cl}]}\end{array}$ & AUC & $\begin{array}{l}C h P^{2} \text { (P value)/ } \\
P^{2}\end{array}$ \\
\hline All cancers & $0.74[0.67-0.80]$ & $0.83[0.75-0.89]$ & 4.45 [2.99-6.60] & $0.31[0.25-0.38]$ & 14.35 [9.13-22.54] & 0.85 & $<0.001 ; 99.61 \%$ \\
\hline \multicolumn{8}{|l|}{ Cancer type } \\
\hline PC vs. non-cancer & $0.73[0.66-0.80]$ & $0.76[0.64-0.85]$ & 3.12 [2.03-4.80] & $0.35[0.27-0.45]$ & 8.92 [5.05-15.77] & 0.80 & $<0.0001 ; 97.62 \%$ \\
\hline PC vs. HC & $0.71[0.66-0.75]$ & $0.66[0.62-0.70]$ & $2.21[1.58-3.11]$ & $0.38[0.25-0.59]$ & 6.32 [2.75-14.52] & 0.73 & $0.001 ; 78.4 \%$ \\
\hline PC vs. Pancreatitis & $0.70[0.63-0.76]$ & $0.66[0.57-0.75]$ & $2.81[1.40-5.64]$ & $0.40[0.28-0.57]$ & $8.86[4.81-16.31]$ & 0.81 & $0.8281 ; 0.0 \%$ \\
\hline $\begin{array}{l}\text { PC (stage I-II) vs. } \\
\text { non-cancer }\end{array}$ & $0.70[0.63-0.75]$ & $0.65[0.61-0.69]$ & 2.04 [1.56-2.68] & $0.40[0.26-0.62]$ & 5.48 [2.58-11.63] & 0.76 & $0.1070 ; 55.2 \%$ \\
\hline $\begin{array}{l}\text { PC (stage III-IV) vs. } \\
\text { non-cancer }\end{array}$ & $0.66[0.56-0.76]$ & $0.74[0.60-0.86]$ & 3.00 [0.82-11.05] & $0.47[0.35-0.62]$ & 6.67 [2.67-16.69] & 0.78 & $0.2984 ; 7.5 \%$ \\
\hline Esophageal carcinoma & $0.47[0.42-0.53]$ & $0.83[0.80-0.87]$ & 3.83 [1.46-10.05] & 0.34 [0.12-0.93] & 13.85 [8.19-23.42] & 0.84 & $0.4586 ; 0.0 \%$ \\
\hline \multicolumn{8}{|l|}{ Sample type } \\
\hline Plasma & $0.69[0.64-0.73]$ & $0.69[0.62-0.75]$ & 2.21 [1.79-2.73] & $0.44[0.35-0.54]$ & 6.09 [4.00-9.28] & 0.78 & $0.5158 ; 0.0 \%$ \\
\hline Serum & $0.67[0.65-0.69]$ & $0.80[0.78-0.81]$ & $5.25[3.16-8.71]$ & $0.30[0.23-0.41]$ & 20.28 [10.12-40.61] & 0.87 & $<0.0001 ; 88.9 \%$ \\
\hline \multicolumn{8}{|l|}{ Cut-off setting } \\
\hline $\begin{array}{l}\geq 2000 \mathrm{pg} / \mathrm{mL} \\
\text { (PC vs. non-cancer) }\end{array}$ & $0.66[0.60-0.71]$ & $0.74[0.65-0.82]$ & $2.46[1.70-3.55]$ & $0.46[0.37-0.57]$ & 6.16 [3.53-10.74] & 0.77 & $0.3172 ; 15.3 \%$ \\
\hline $\begin{array}{l}<2000 \mathrm{pg} / \mathrm{mL} \text { (PC vs. } \\
\text { non-cancer) }\end{array}$ & $0.69[0.66-0.71]$ & $0.73[0.70-0.75]$ & 3.30 [1.84-5.93] & $0.35[0.26-0.47]$ & 11.98 [4.42-32.49] & 0.85 & $<0.0001 ; 90.4 \%$ \\
\hline $\begin{array}{l}\geq 2000 \mathrm{pg} / \mathrm{mL} \\
\text { (all cancers vs. } \\
\text { non-cancer) }\end{array}$ & $0.65[0.60-0.69]$ & $0.80[0.73-0.85]$ & $2.91[1.94-4.37]$ & $0.45[0.39-0.52]$ & 7.34 [4.52-11.91] & 0.77 & $0.2465 ; 25.0 \%$ \\
\hline $\begin{array}{l}<2000 \mathrm{pg} / \mathrm{mL} \\
\text { (all cancers vs. } \\
\text { non-cancer) }\end{array}$ & $0.68[0.66-0.70]$ & $0.78[0.76-0.80]$ & $4.36[2.77-6.85]$ & $0.29[0.22-0.40]$ & $17.79[9.00-35.18]$ & 0.87 & $<0.0001 ; 88.1 \%$ \\
\hline \multicolumn{8}{|l|}{ Ethnicity } \\
\hline Caucasian & $0.75[0.71-0.78]$ & $0.71[0.68-0.75]$ & $2.67[2.10-3.41]$ & $0.32[0.25-0.41]$ & 9.94 [6.57-15.04] & 0.83 & $0.0335 ; 46.4 \%$ \\
\hline Asian & $0.65[0.63-0.67]$ & $0.81[0.79-0.83]$ & 7.47 [3.21-17.37] & $0.36[0.26-0.52]$ & $22.01[7.65-63.31]$ & 0.83 & $<0.0001 ; 93.7 \%$ \\
\hline
\end{tabular}

Abbreviations: PLR positive likelihood ratio, NLR negative likelihood ratio, DOR diagnostic odds ratio, AUC area under the curve, MT-PCR multiplexed tandem PCR, QUADAS Quality Assessment for Studies of Diagnostic Accuracy, EC esophageal carcinoma, $H C$ healthy control, $P C$ pancreatic cancer, $A U C$ area under the curve

alternative biomarker to differentiate patients with DST from those with non-cancerous tumors.

Stratified analyses were performed in the diagnostic meta-analysis based on cancer type, sample type, cut-off setting, and ethnicity. As summarized in Table 3, the pooled AUC of GDF-15 to rule out PC, EC, GC, and liver cancer were estimated to be $0.82,0.84,0.90$, and 0.85 , respectively. Moreover, GDF-15 had an AUC of 0.82 for its ability to distinguish $\mathrm{PC}$ from pancreatitis, which was a higher value than the AUC for its ability to distinguish PC from healthy individuals $(\mathrm{AUC}=0.73$ ). When meta-analyzed based on sample type, serum-based GDF-15 testing achieved a specificity of 0.80 (95\%CI: $0.78-0.81$ ) and an AUC of 0.87 , which were superior to plasma-based analysis. We found differences in diagnostic efficacy based on cut-off value: a cut-off setting $<2000 \mathrm{pg} / \mathrm{mL}$ showed an AUC of 0.85 for PC (PC vs. non-cancerous tumors), and 0.87 for all cancers (all cancers vs. non-cancerous tumors). In the meta-analysis based on ethnicity, GDF-15 testing in Caucasian and Asian patients yielded an AUC of 0.83, whereas the Asian-based test conferred a higher specificity of 0.81 (95\% CI: 0.79-0.83). The raw data used for the diagnostic meta-analysis was attached as Additional file 3.

\section{Prognostic significance}

Analysis of a 2200 patient cohort was used to define the association between GDF-15 levels and patient prognosis. Patients with DST who had increased GDF-15 levels had worse overall survival $(\mathrm{OS})(\mathrm{HR}=2.34,95 \% \mathrm{CI}$ : 2.03-2.70, $\left.P<0.001 ; \mathrm{I}^{2}=0.0 \%\right)$ compared with patients with low GDF-15 levels (Fig. 3a). Moreover, elevated levels of GDF-15 were associated with a significantly shorter OS time in patients with $\mathrm{CRC}(\mathrm{HR}=2.27$, 95\%CI: 1.96-2.63, $P<0.001 ; \mathrm{I}^{2}=0.0 \%$ ) (Fig. 3b). We also included 6 individual data sets for CSS and TSS, and the results showed that GDF-15 levels were correlated with worse CSS in CRC ( $\mathrm{HR}=2.33$, 95\% CI: $1.95-$ 2.78, $P<0.001 ; \mathrm{I}^{2}=0.0 \%$ )(Fig. 3c). The raw data used for the prognostic meta-analysis was attached as Additional file 4. 


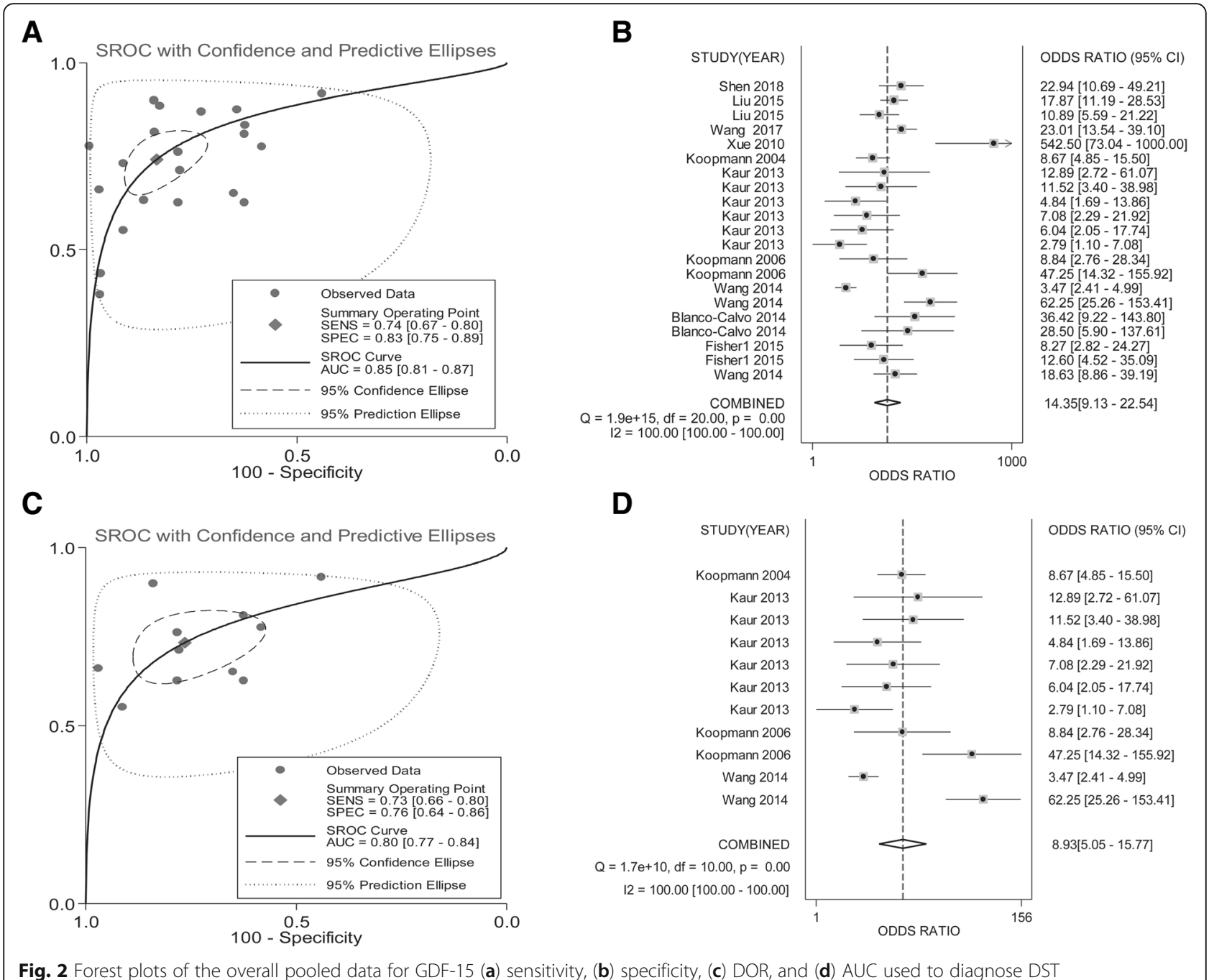

Fig. 2 Forest plots of the overall pooled data for GDF-15 (a) sensitivity, (b) specificity, (c) DOR, and (d) AUC used to diagnose DST

\section{Influence analysis and meta-regression}

Influence analysis was conducted for both diagnostic and prognostic meta-analyses using STATA 12.0 software. One individual study [16] was identified as an outlier in the overall pooled diagnostic dataset for DST (Fig. 4a) and PC (Fig. 4b). However, no outlier studies were found at the upper or lower CI limit of the prognostic studies, indicating that the selected studies had relatively high homogeneity (Fig. 4c, d, and e).

Meta-regression was performed to trace the causes of heterogeneity, wherein seven covariates, comprising ethnicity, sample size, control size, cancer type, test matrix, cut-off setting, and QUADAS score, were predefined. As displayed in Additional file 5, the analysis of QUADAS score received the lowest $P$-value (0.0349) among the analyses, suggesting that QUADAS score is the likely source of heterogeneity among diagnostic studies.

\section{Publication bias}

Publication bias analysis, assessed by Deeks' funnel plot asymmetry test, demonstrated no clear bias in the overall diagnostic meta-analyses of DST and PC (Fig. 5a and b, $n=22$ or $12, P=0.375$ or 0.479$)$. Additionally, no significant publication bias, as assessed using Egger's and Begg's tests, was detected in the meta-analyzed prognostic data (all with $P>0.05$ ) (Fig. 5c, d and e).

\section{Discussion}

Expression levels of growth differentiation factor 15 (GDF-15) are increased in most DSTs, including those of the colon [27], stomach [28], pancreas [29], liver [23, 24], and esophagus [21, 22]. Given the important role of GDF-15 in DST, GDF-15 has the potential to be a promising biomarker in DST $[6,8-19,21-24]$. Nevertheless, the utility of GDF-15 as a biomarker has not been confirmed due to a lack of data supported by evidence-based 


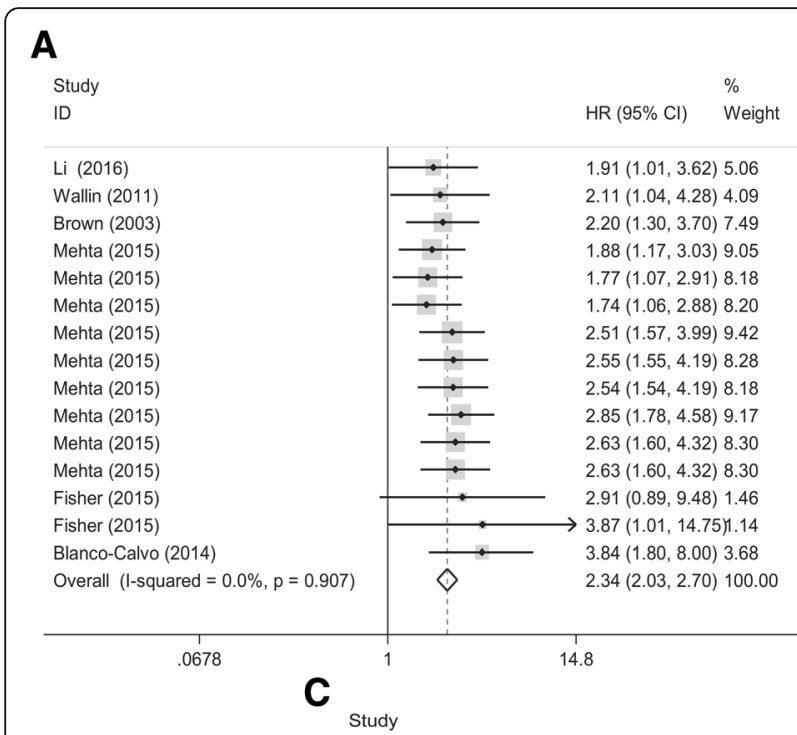

ID
B Study $\%$ ID $\quad H R(95 \%$ Cl) Weight

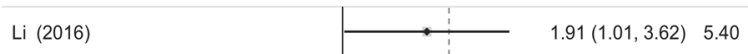
Wallin (2011) $2.11(1.04,4.28) \quad 4.37$ Brown (2003) Mehta (2015) Mehta (2015) Mehta (2015) Mehta (2015) Mehta (2015) Mehta (2015) Mehta (2015) Mehta (2015) Mehta (2015) Overall $(I-$ squared $=0.0 \%, p=0.925$ ) \begin{tabular}{c|c:c} 
& & \\
& & \\
\hline 1 & 1 & 4.58
\end{tabular} $\%$ $\mathrm{HR}(95 \% \mathrm{Cl}) \quad$ Weight

$1.94(1.12,3.37) \quad 10.24$ $1.65(0.92,2.94) \quad 9.21$ $1.67(0.93,3.00) \quad 9.06$ $2.64(1.54,4.51) \quad 10.76$ $2.60(1.45,4.67) \quad 9.09$ $2.67(1.49,4.80) \quad 9.08$ $2.73(1.57,4.75) \quad 10.14$ $2.34(1.30,4.21) \quad 9.00$ $2.40(1.33,4.34) \quad 8.88$ $2.92(1.56,5.45) \quad 7.95$

Mehta (2015)

Wang (2017)

Wang (2017)

Overall (I-squared $=0.0 \%, p=0.922$ )

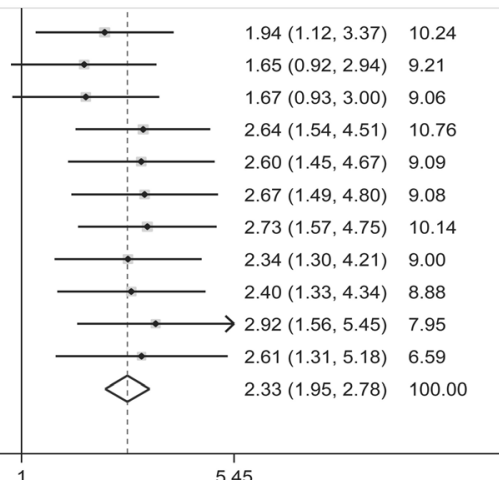

183

5.45

Fig. 3 Forest plots of pooled HRs (95\% Cl) for GDF-15 levels in the prognostic datasets. a Pooled HR (95\% Cl) of OS data for DST; b pooled HR $(95 \% \mathrm{Cl})$ of OS data for CRC; c pooled HR $(95 \% \mathrm{Cl})$ of CSS/TSS data for CRC

medicine. In the present study, we performed a meta-analysis using recent information obtained regarding GDF-15 as a diagnostic and prognostic biomarker in DSTs.

As expected, GDF-15 was used successfully as a diagnostic biomarker in DST: the pooled sensitivity, specificity, and AUC for the discriminative performance of GDF-15 to rule out DST were $0.74,0.83$, and 0.84 , respectively. Although the combined sensitivity was not significantly high, the specificity and AUC were relatively high as well, and illustrated an acceptable diagnostic performance for GDF-15. The diagnostic odds ratio (DOR) is another measure of diagnostic effectiveness, with a value higher than 1.0 representing diagnostic validity [30]. Herein, we obtained a DOR of 14.07, further suggesting that GDF-15 testing can be used to diagnose DST. The pooled PLR of 4.38 also indicated that GDF-15 testing harbored a ratio between the true-positive and false-positive rate.

Several groups have demonstrated that GDF-15 may be used as a biomarker to assist in the detection of PC,
EC, GC, and HCC [12, 13, 15-19, 21-24]. In our stratified analysis, 4 groups of carcinomas had been evaluated repeatedly: the pooled AUC of GDF-15 to rule out PC, $\mathrm{EC}, \mathrm{GC}$, and HCC were estimated to be $0.82,0.84,0.90$, and 0.85 , respectively, showing that GDF-15 testing achieved a significant level of efficacy in confirming GC. In PC, GDF-15 testing had an AUC of 0.82 for its ability to differentiate PC from pancreatitis, which was higher than its ability to distinguish PC from healthy individuals. These data indicate that GDF-15 may also be a useful indicator for the differential diagnosis of $\mathrm{PC}$ and pancreatitis. Additionally, we observed matrix effects for the test performance: serum-based GDF-15 testing yielded a better AUC than that for plasma-based analysis, suggesting that serum samples may be more suitable than plasma samples for GDF-15 testing. We also found differences in diagnostic efficacy based on cut-off value: a cut-off setting of less than $2000 \mathrm{pg} / \mathrm{mL}$ exhibited better performance for all cancer types. Lastly, for data stratified by ethnicity, we found an equal diagnostic 

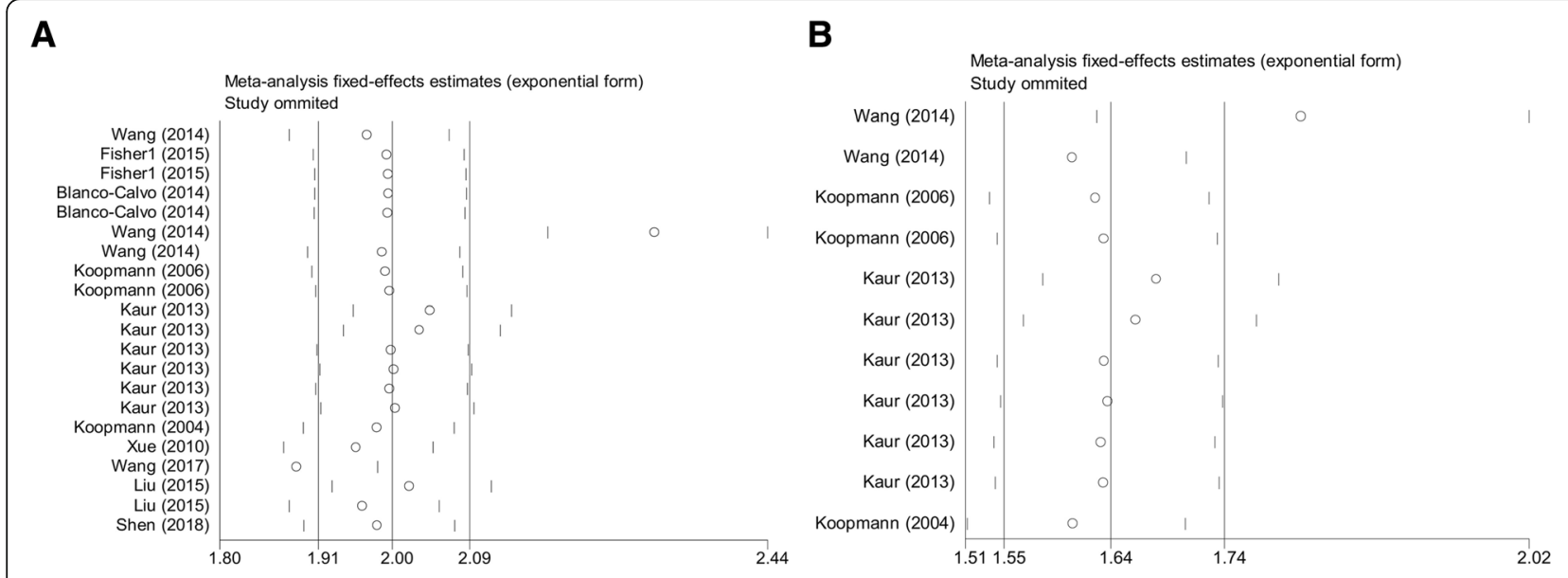

C

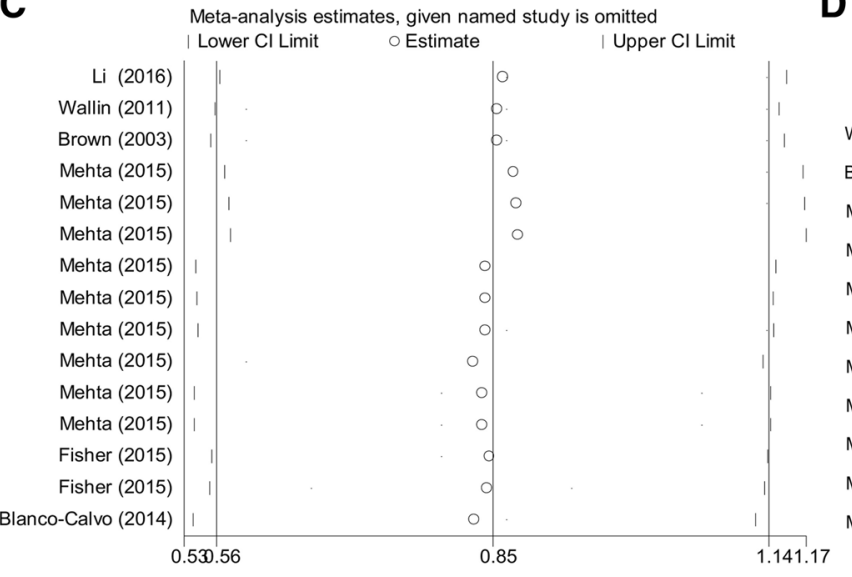

D

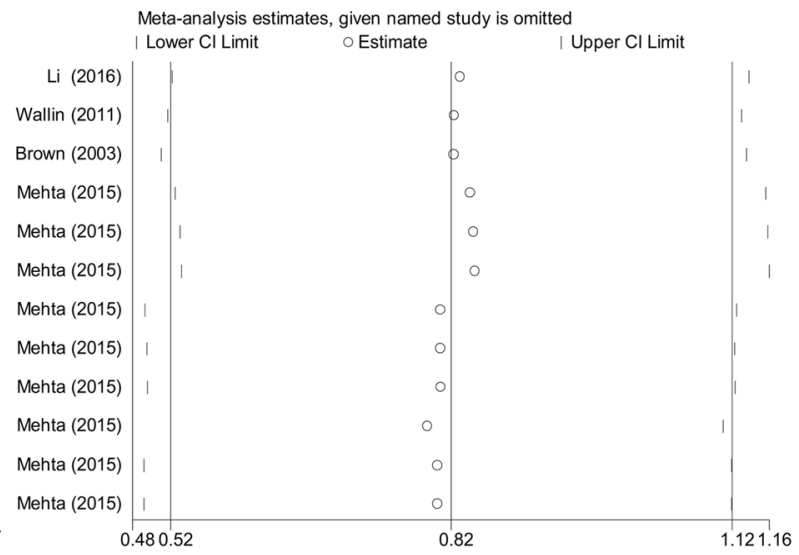

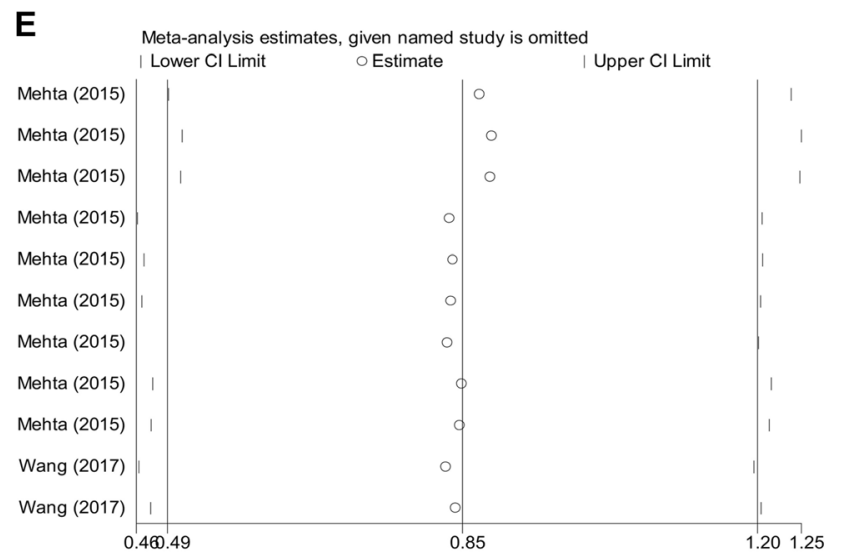

Fig. 4 Influence analysis of outlier studies. a The overall pooled diagnostic dataset for DST; $\mathbf{b}$ the combined diagnostic dataset for GDF-15 levels in PC; (c) the overall prognostic dataset of OS for DST; $\mathbf{d}$ the combined prognostic dataset of OS, and CSS (e) for GDF-15 in CRC

efficacy of GDF-15 testing between Caucasians and Asians. However, without additional data to support these findings, more investigation is needed.

We found that increased levels of GDF-15 were an independent prognostic marker for DST [8-11, 13-15, 21, 22]. Previously, the topic of whether GDF-15 could serve as prognostic markers for OS, DFS, or RFS in cancer was considered controversial. In our prognostic analysis, 2106 patients with complete follow-up data were included. A clear association between increased GDF-15 levels and shorter OS was observed in patients with DST (HR = $2.34)$, as well as in colorectal cancer $(\mathrm{HR}=2.27)$. We also 


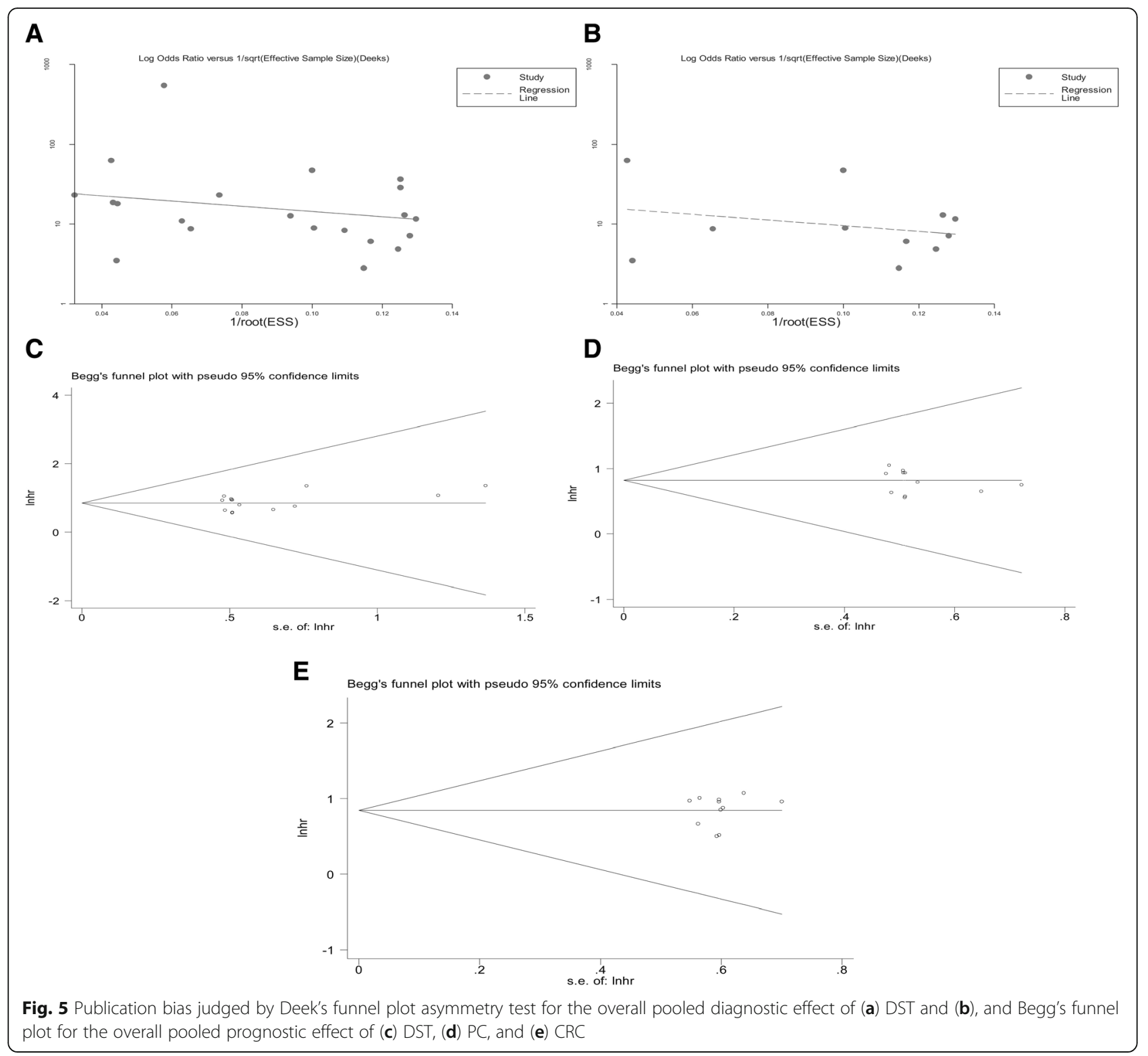

included 11 individual studies that measured CSS or TSS in CRC, with results that showed a correlation between GDF-15 expression and poor CSS and TSS $(\mathrm{HR}=2.33)$. These data suggest that GDF-15 could be used as an independent prognostic biomarker in DST. Previous studies have hypothesized that GDF-15 could be used to assist the prediction of cancer recurrence and metastasis in CRC [31, 32]. However, the data obtained for CRC recurrence and metastasis were not sufficient for our study, and were therefore not analyzed.

Study heterogeneity and bias are very common in meta-analysis studies [33]. We observed significant heterogeneity in our diagnostic meta-analyses; thus, we attempted to interpret the cause of this heterogeneity. Firstly, we included studies that included varying patient population. Secondly, patients participating in these studies had different types of cancer and received a wide range of treatments. Moreover, the primary method of GDF-15 expression detection testing (ELISA) used a different cut-off value in each study, particularly that the cut-off points were obviously higher in gastric and liver cancers than other malignancies. Whether the differences in cut-off points were due to cancer type or limited studies still warranted further investigations. Collectively, these factors above may have resulted in non-homogeneous conditions. We therefore conducted sensitivity analysis and meta-regression test. Our sensitivity analysis identified one outlier study, and the degree of heterogeneity was decreased after we excluded all outlier data from the analysis. The univariate meta-regression test 
showed that only study quality (different QUADAS scores) seemed to be a source of heterogeneity among all other studies.

Limitations of this study include low sample sizes for some cancer types and few available current articles. Secondly, significant heterogeneity was observed in the diagnostic meta-analysis, compromising the overall study accuracy. Lastly, the method used to detect GDF-15 expression consisted primarily of ELISA, which might not be the optimal method to detect GDF-15.

\section{Conclusions}

In summary, we meta-analyzed the diagnostic and prognostic value of GDF-15 in patients with DST. Our analysis provides evidence that elevated GDF-15 levels may be used as a novel diagnostic and prognostic biomarker for DST.

\section{Additional files}

Additional file 1: Table S1. Study quality of the diagnostic studies, as assessed by the 14-item QUADAS tool. (DOC $53 \mathrm{~kb}$ )

Additional file 2: Table S2. Evaluation of bias in the retrospective cohort studies, as assessed using the Newcastle-Ottawa Scale (NOS) checklist. (DOC $36 \mathrm{~kb}$ )

Additional file 3: Table S3. Raw data used for the diagnostic metaanalysis. The numerical values of TP (true positive), FP (false positive), FN (false negative) and TN (true negative) are available, and were used to construct the $2 \times 2$ table. (DOC $74 \mathrm{~kb}$ )

Additional file 4: Table S4. Raw data used for the prognostic metaanalysis. Single HR with $95 \% \mathrm{Cl}$ was extracted or calculated form original studies, and the pooled $\mathrm{HR}$ with $95 \% \mathrm{Cl}$ was generated. (DOC $74 \mathrm{~kb}$ )

Additional file 5: Table S5. Meta-regression test of the overall diagnostic analysis based on different covariates. (DOC 29 kb)

\section{Abbreviations}

AUC: Area under the curve; CRC: Colorectal cancer; CSS: Cancer-specific survival; DFS: Disease free survival; DOR: Diagnostic odds ratio; DST: Digestive system tumors; EC: Esophageal carcinoma; ELISA: Enzyme linked immunosorbent assay; GC: Gastrointestinal cancer; GDF-15: Cytokine growth differentiation factor 15; HC: Healthy control; HCC: Hepatocellular carcinoma; HR: Hazard ratio; MeSH: Medical Subject Headings; MIC-1: Macrophage inhibitory cytokine-1; MT-PCR: Multiplexed tandem PCR; NLR: Negative likelihood ratio; NOS: Newcastle-Ottawa Scale; OS: Overall survival; PC: Pancreatic cancer; PFS: Progression-free survival; PLR: Positive likelihood ratio; QUADAS: Quality assessment for studies of diagnostic accuracy; RFS: Recurrence-free survival; TGF- $\beta 1$ : Transforming growth factor- $\beta$; TSS: Tumor-specific survival

\section{Acknowledgements}

None

\section{Funding}

None

\section{Availability of data and materials}

All data generated or analyzed during this study are included in this published article and its Additional file 3 and Additional file 4.

\section{Authors' contributions}

Conceived and designed the experiments: YQW; Data extraction: MYJ and SJG; Analyzed the data: TJ; Wrote the paper: YQW. All authors read and approved the final manuscript.
Ethics approval and consent to participate

Not applicable

\section{Consent for publication}

Not applicable

Competing interests

The authors declare that they have no competing interests.

\section{Publisher's Note}

Springer Nature remains neutral with regard to jurisdictional claims in published maps and institutional affiliations.

\section{Author details}

${ }^{1}$ Department of Clinical Laboratory, The First Affiliated Hospital, and College of Clinical Medicine of Henan University of Science and Technology, No.24 Jinghua Road, Jianxi District, Luoyang 471000, China. ²Department of Rehabilitation, Seafarers' General Hospital in Heilongjiang Province, Harbin 150000, China. ' Department of Clinical Laboratory, The Fifth People's Hospital of Haimen City in Jiangsu Province, Haimen 226131, China.

Received: 5 September 2018 Accepted: 19 February 2019

Published online: 26 February 2019

References

1. Siegel RL, Miller KD, Jemal A. Cancer statistics, 2016. CA Cancer J Clin. 2016; 66:7-30.

2. Miller KD, Siegel RL, Lin CC, Mariotto AB, Kramer JL, Rowland JH, Stein KD, Alteri R, Jemal A. Cancer treatment and survivorship statistics, 2016. CA Cancer J Clin. 2016;66:271-89.

3. Bauskin AR, Jiang L, Luo XW, Wu L, Brown DA, Breit SN. The TGF-beta superfamily cytokine MIC-1/GDF15: secretory mechanisms facilitate creation of latent stromal stores. J Interf Cytokine Res. 2010;30:389-97.

4. Bootcov MR, Bauskin AR, Valenzuela SM, Moore AG, Bansal M, He XY, Zhang HP, Donnellan M, Mahler S, Pryor K, et al. MIC-1, a novel macrophage inhibitory cytokine, is a divergent member of the TGF-beta superfamily. Proc Natl Acad Sci U S A. 1997;94:11514-9.

5. Adela R, Banerjee SK. GDF-15 as a target and biomarker for diabetes and cardiovascular diseases: a translational prospective. J Diabetes Res. 2015; 2015:490842.

6. Karan D, Holzbeierlein J, Thrasher JB. Macrophage inhibitory cytokine-1: possible bridge molecule of inflammation and prostate cancer. Cancer Res. 2009;69:2-5

7. Wollert KC, Kempf T, Wallentin L. Growth differentiation factor 15 as a biomarker in cardiovascular disease. Clin Chem. 2017;63:140-51.

8. Li C, Wang X, Casal I, Wang J, Li P, Zhang W, Xu E, Lai M, Zhang H. Growth differentiation factor 15 is a promising diagnostic and prognostic biomarker in colorectal cancer. J Cell Mol Med. 2016;20:1420-6.

9. Wallin U, Glimelius B, Jirstrom K, Darmanis S, Nong RY, Ponten F, Johansson C, Pahlman L, Birgisson H. Growth differentiation factor 15: a prognostic marker for recurrence in colorectal cancer. Br J Cancer. 2011;104:1619-27.

10. Brown DA, Ward RL, Buckhaults P, Liu T, Romans KE, Hawkins NJ, Bauskin AR, Kinzler KW, Vogelstein B, Breit SN. MIC-1 serum level and genotype: associations with progress and prognosis of colorectal carcinoma. Clin Cancer Res. 2003;9:2642-50.

11. Mehta RS, Chong DQ, Song M, Meyerhardt JA, Ng K, Nishihara R, Qian Z, Morikawa T, Wu K, Giovannucci EL, et al. Association between plasma levels of macrophage inhibitory Cytokine-1 before diagnosis of colorectal Cancer and mortality. Gastroenterology. 2015;149:614-22.

12. Xue H, Lu B, Zhang J, Wu M, Huang Q, Wu Q, Sheng H, Wu D, Hu J, Lai M. Identification of serum biomarkers for colorectal cancer metastasis using a differential secretome approach. J Proteome Res. 2010;9:545-55.

13. Wang X, Yang Z, Tian H, Li Y, Li M, Zhao W, Zhang C, Wang T, Liu J, Zhang A, et al. Circulating MIC-1/GDF15 is a complementary screening biomarker with CEA and correlates with liver metastasis and poor survival in colorectal cancer. Oncotarget. 2017;8:24892-901.

14. Skipworth RJ, Deans DA, Tan BH, Sangster K, Paterson-Brown S, Brown DA, Hunter M, Breit SN, Ross JA, Fearon KC. Plasma MIC-1 correlates with systemic inflammation but is not an independent determinant of nutritional status or survival in oesophago-gastric cancer. Br J Cancer. 2010;102:665-72. 
15. Blanco-Calvo M, Tarrio N, Reboredo M, Haz-Conde M, Garcia J, Quindos M, Figueroa A, Anton-Aparicio L, Calvo L, Valladares-Ayerbes M. Circulating levels of GDF15, MMP7 and miR-200c as a poor prognostic signature in gastric cancer. Future Oncol. 2014;10:1187-202.

16. Wang X, Li Y, Tian H, Qi J, Li M, Fu C, Wu F, Wang Y, Cheng D, Zhao W, et al. Macrophage inhibitory cytokine 1 (MIC-1/GDF15) as a novel diagnostic serum biomarker in pancreatic ductal adenocarcinoma. BMC Cancer. 2014; 14:578.

17. Koopmann J, Rosenzweig CN, Zhang Z, Canto Ml, Brown DA, Hunter M, Yeo C, Chan DW, Breit SN, Goggins M. Serum markers in patients with resectable pancreatic adenocarcinoma: macrophage inhibitory cytokine 1 versus CA19-9. Clin Cancer Res. 2006;12:442-6.

18. Kaur S, Chakraborty S, Baine MJ, Mallya K, Smith LM, Sasson A, Brand R, Guha S, Jain M, Wittel U, et al. Potentials of plasma NGAL and MIC-1 as biomarker(s) in the diagnosis of lethal pancreatic cancer. PLoS One. 2013;8: e55171.

19. Koopmann J, Buckhaults P, Brown DA, Zahurak ML, Sato N, Fukushima N, Sokoll LJ, Chan DW, Yeo CJ, Hruban RH, et al. Serum macrophage inhibitory cytokine 1 as a marker of pancreatic and other periampullary cancers. Clin Cancer Res. 2004;10:2386-92.

20. Hogendorf P, Durczynski A, Skulimowski A, Kumor A, Poznanska G, Strzelczyk J. Growth differentiation factor (GDF-15) concentration combined with Ca125 levels in serum is superior to commonly used cancer biomarkers in differentiation of pancreatic mass. Cancer Biomark. 2018;21: $505-11$

21. Wang XB, Jiang XR, Yu XY, Wang $L$, He S, Feng FY, Guo LP, Jiang W, Lu SH. Macrophage inhibitory factor 1 acts as a potential biomarker in patients with esophageal squamous cell carcinoma and is a target for antibodybased therapy. Cancer Sci. 2014;105:176-85.

22. Fisher OM, Levert-Mignon AJ, Lord SJ, Lee-Ng KK, Botelho NK, Falkenback D, Thomas ML, Bobryshev YV, Whiteman DC, Brown DA, et al. MIC-1/GDF15 in Barrett's oesophagus and oesophageal adenocarcinoma. Br J Cancer. 2015; 112:1384-91.

23. Liu X, Chi X, Gong Q, Gao L, Niu Y, Chi X, Cheng M, Si Y, Wang M, Zhong J, et al. Association of serum level of growth differentiation factor 15 with liver cirrhosis and hepatocellular carcinoma. PLoS One. 2015;10:e0127518.

24. Shen $Y$, Zhang ZB, Wu SD, Wu XB, Li J. Research on values of GDF-15 level in the diagnosis of primary liver cancer and evaluation of chemotherapeutic effect. Eur Rev Med Pharmacol Sci. 2018;22:3749-54.

25. Whiting P, Rutjes AW, Reitsma JB, Bossuyt PM, Kleijnen J. The development of QUADAS: a tool for the quality assessment of studies of diagnostic accuracy included in systematic reviews. BMC Med Res Methodol. 2003;3:25.

26. Stang A. Critical evaluation of the Newcastle-Ottawa scale for the assessment of the quality of nonrandomized studies in meta-analyses. Eur J Epidemiol. 2010;25:603-5.

27. Khaled YS, Elkord E, Ammori BJ. Macrophage inhibitory cytokine-1: a review of its pleiotropic actions in cancer. Cancer Biomark. 2012;11:183-90.

28. Han M, Dai D, Yousafzai NA, Wang F, Wang H, Zhou Q, Lu H, Xu W, Feng L, Jin H, Wang X. CXXC4 activates apoptosis through up-regulating GDF15 in gastric cancer. Oncotarget. 2017:8:103557-67.

29. Loosen SH, Neumann UP, Trautwein C, Roderburg C, Luedde T. Current and future biomarkers for pancreatic adenocarcinoma. Tumour Biol. 2017;39: 1010428317692231.

30. Jones CM, Ashrafian H, Darzi A, Athanasiou T. Guidelines for diagnostic tests and diagnostic accuracy in surgical research. J Investig Surg. 2010;23:57-65.

31. Vocka M, Langer D, Fryba V, Petrtyl J, Hanus T, Kalousova M, Zima T, Petruzelka L. Growth/differentiation factor 15 (GDF-15) as new potential serum marker in patients with metastatic colorectal cancer. Cancer Biomark. 2018;21:869-74.

32. Brown DA, Hance KW, Rogers CJ, Sansbury LB, Albert PS, Murphy G, Laiyemo AO, Wang Z, Cross AJ, Schatzkin A, et al. Serum macrophage inhibitory cytokine-1 (MIC-1/GDF15): a potential screening tool for the prevention of colon cancer? Cancer Epidemiol Biomark Prev. 2012;21:337-46.

33. Ioannidis JP. Interpretation of tests of heterogeneity and bias in metaanalysis. J Eval Clin Pract. 2008:14:951-7.

\section{Ready to submit your research? Choose BMC and benefit from:}

- fast, convenient online submission

- thorough peer review by experienced researchers in your field

- rapid publication on acceptance

- support for research data, including large and complex data types

- gold Open Access which fosters wider collaboration and increased citations

- maximum visibility for your research: over $100 \mathrm{M}$ website views per year

At BMC, research is always in progress.

Learn more biomedcentral.com/submissions 